\title{
MICROBIOLOGICAL STUDY OF VARIOUS CANDIDA SPECIES AND ITS ANTIFUNGAL SENSITIVITY TESTING ISOLATED FROM ANTENATAL WOMEN WITH VAGINITIS, IN TERTIARY CARE TEACHING HOSPITAL, WESTERN INDIA
}

\author{
PANCHAL P.A. ${ }^{*}{ }^{*}$, KATARA R.K. ${ }^{1}$, MEHTA R.C. ${ }^{2}$, SONI S.T. ${ }^{1}$, NANERA A. ${ }^{1}$, TRIVEDI N.A. ${ }^{1}$, DESAI K.J. ${ }^{3}$ AND \\ VEGAD M.M.1
}

1Department of Microbiology, B.J. Medical College, Ahmedabad- 380016, Gujarat, India.

2Department of Pathology, B.J. Medical College, Ahmedabad- 380016, Gujarat, India.

${ }^{3}$ Department of Microbiology, Government Medical College, Bhavnagar- 364001, Gujarat, India.

${ }^{*}$ Corresponding Author: Email- piyushpanchal26@yahoo.com

Received: September 20, 2013; Accepted: October 07, 2013

\begin{abstract}
-
Background and Objective: An estimated $75 \%$ of women will have at least one episode of VVC, and 40-45\% will have two or more episodes within their lifetime and infection occurs more frequently in pregnant women-CDC. This study was conducted to estimate the prevalence of vulvovaginal candidiasis along with its antifungal susceptibility pattern among antenatal women attending Obstetric and Gynecology Department Sir T Hospital, Bhavnagar, Gujarat, India.

Methods: Vaginal swab specimens for culture were drawn from 100 female attending obstetric and gynecology Department, Sir T Hospital, Bhavnagar during August 2009 to August 2011. The specimen was inoculated into Sabouraud's dextrose agar and the isolated colonies were identified by battery of biochemical reactions. The specimen were also inoculated Chrom Candida differential agar. Antifungal sensitivity pattern of isolates was studied by Modified Kirby Bauer Disc diffusion technique on Muller Hinton agar with $2 \%$ glucose and Methylene blue.

Result: In this study, a total number of 100 patients of vaginal Candidiasis were included, among them 40 samples indicates positivity, out of them 22 samples (55\%) indicate Candida albicans and 18 samples (45\%) are Non albicans species.

Conclusion: Vaginal candidiasis is an extremely common infection in $60-70 \%$ women during their reproductive age at least once in their lives. There is an increase in infections with non-albicans Candida spp. and few of them are intrinsically resistant to azoles. Effective antifungal treatment is an important criterion in treating the candidial infections.
\end{abstract}

Keywords- Candida, vaginitis, pregnancy, chrom agar, antifungal susceptibility

Citation: Panchal P.A., et al. (2013) Microbiological Study of Various Candida Species and Its Antifungal Sensitivity Testing Isolated from Antenatal Women with Vaginitis, in Tertiary Care Teaching Hospital, Western India. International Journal of Microbiology Research, ISSN: 09755276 \& E-ISSN: 0975-9174, Volume 5, Issue 6, pp.-486-489.

Copyright: Copyright@2013 Panchal P.A., et al. This is an open-access article distributed under the terms of the Creative Commons Attribution License, which permits unrestricted use, distribution and reproduction in any medium, provided the original author and source are credited.

\section{Introduction}

Candida species are the second most common cause of vulvovaginitis worldwide [1]. The prevalence of vulvovaginal candidiasis (VVC) is increasing due to the extensive utilization of broadspectrum antibiotics as well as increased cases of immunocompromised patients [2,3]. An estimated $75 \%$ of women will have at least one episode of VVC, and $40-45 \%$ will have two or more episodes within their lifetime and infection occurs more frequently in pregnant women. The three diseases most frequently associated with vaginal discharge are Bacterial Vaginosis, trichomoniasis and candidiasis. The subjective symptoms may be leucorrhoea typically curdy white discharge itching over vulva, burning micturition backache and/or dyspareunia [4]. As Vulvovaginal candidiasis is so common in women during their childbearing years, it is important to understand the pathology of this disease as well as the safety or risks of drugs used to treat it during pregnancy. The incidence of yeast infection also rise in immune compromised patient due to disease like diabetes, sexual transmitted disease, endocrine disorder \& leukemia also. Cure rate is $80 \%$ for uncomplicated cases. Approximately 10 $20 \%$ of women will have complicated VVC that necessitates diagnostic and therapeutic considerations. The complications can be treatment failure in about $20 \%$. Recurrent candidiasis can affect up to $50 \%$ of sufferers at a given period in their life, depression and psychosexual problems can occur in women who suffer recurrent episodes \& treatment during pregnancy is more likely to fail.

This study was conducted to estimate the prevalence of vulvovaginal candidiasis along with its antifungal susceptibility pattern among antenatal women attending Obstetric and Gynecology Department, Sir T Hospital, Bhavnagar, Gujarat, India.

\section{Materials and Methods}

In this study antenatal women who were attending Obstetric and 
Gynecology Department, Sir T Hospital, Bhavnagar, Gujarat, India in duration of August 2009 to August 2011 with complaints of discharge per vaginam were included. On per vaginam and per speculum examination, from the women with curdy white discharge Vaginal swabs were taken. 100 vaginal swabs were taken \& were inoculated into Sabouraud's Dextrose Agar and Hichrom Candida agar $[5,6]$. The White to cream colored, pasty, smooth colonies were identified and confirmed by wet mount, Gram's staining, Germ tube testing and Dalmau plate (cornmeal agar) morphology, Sugar assimilation and fermentation tests. The isolates identified by biochemical tests were also compared with growth and color changes in Hichrom Candida agar. Antifungal sensitivity pattern of isolates were performed by Modified Kirby Bauer Disc diffusion technique on Muller Hinton agar $+2 \%$ glucose + Methylene blue [7]

\section{Ethics}

The permission for this study was taken from Institutional Ethical Committee of Government Medical College, Bhavnagar, Gujarat, India.

\section{Results}

In this study, 100 women of vaginitis were included. The vaginal swabs were processed for fungal culture, among which 45 swabs showed positive yeast like growth. Among them 45 samples indicates positivity, out of them 31 samples indicate Candida albicans and other are non-albicans species. Thus out of total 45 Candida positive specimens $31(68.88 \%)$ specimens were of Candida albicans, 1 was of $(2.22 \%)$ Candida parapsilosis, 8 was of Candida glabrata $(17.77 \%), 2$ was of Candida krusie $(4.4 \%)$, Candia tropicalis was $3(6.66 \%)$ [Table-1].

Table 1- Age distribution within total samples

\begin{tabular}{|ccc|}
\hline Age in Years & Total specimen & Candida isolated \\
\hline $21-30$ & 59 & 35 \\
$31-40$ & 33 & 6 \\
$41-45$ & 8 & 5 \\
Total & 100 & 40 \\
\hline
\end{tabular}

Most of the women from age group 21-30 i.e. $n=59$ and among these women vulvovaginal candidiasis was common and specimen collected showed the maximum positivity rate among these $60 \%$ of the women had curdy discharge and most of the isolated Candida were from this type of discharge [Fig-1], [Table-2], [Fig-2]. Candida albicans found $77 \%$ positivity and other species in another $23 \%$ positivity rate [Table-3].

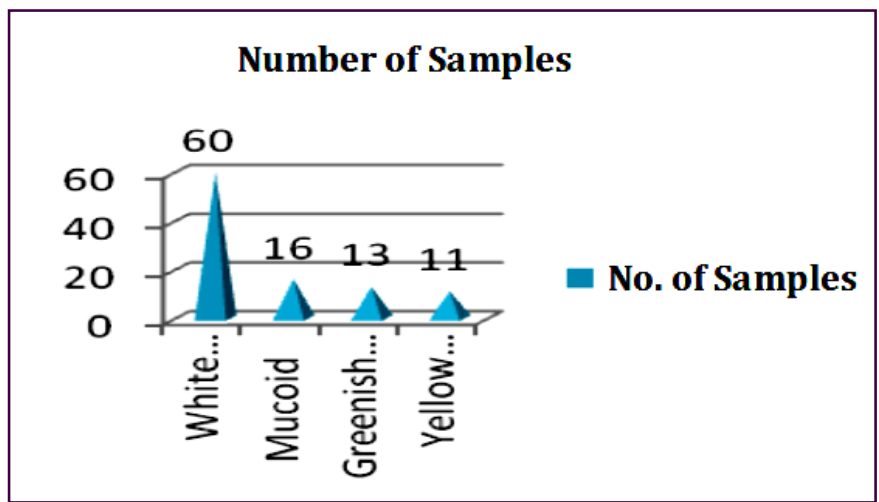

Fig. 1- Types of vaginal discharges in study antenatal women who were attending Obstetric and Gynecology Department Sir T Hospital, Bhavnagar, Gujarat, India
Table 2- Total sample distribution

\begin{tabular}{|lccc|}
$\begin{array}{l}\text { Total no. of } \\
\text { Specimen }\end{array}$ & $\begin{array}{c}\text { Growth obtained in } \\
\text { Saboraud's Dextrose } \\
\text { Agar }\end{array}$ & $\begin{array}{c}\text { Growth on } \\
\text { Hichrom Candida } \\
\text { Agar }\end{array}$ & $\begin{array}{c}\text { No growth on } \\
\text { Saboraud's Dextrose } \\
\text { Agar }\end{array}$ \\
\hline 100 & 40 & 45 & 60 \\
\hline
\end{tabular}

Percentage of positive Candida spp.

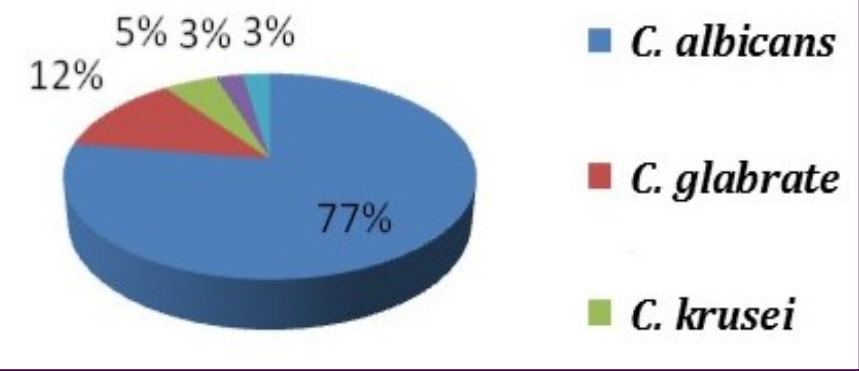

Fig. 2- Isolation rate of various Candida spp.

Table 3- Distribution of different species of isolated Candida

\begin{tabular}{lcc} 
Candida species & $\begin{array}{c}\text { No. of Candida positive } \\
\text { specimen (total 40) }\end{array}$ & $\begin{array}{c}\text { Hichrom candida agar } \\
\text { positive specimen }\end{array}$ \\
\hline Candida albicans & 31 & 31 \\
Candida glabrata & 5 & 8 \\
Candida krusei & 2 & 2 \\
Candia tropicalis & 1 & 3 \\
Candida parapsilosis & 1 & 1 \\
\hline
\end{tabular}

In this study Hichrom agar positivity rate was found more as compared to conventional method of fungal culture methods for isolation, so Hichrom agar is very effective media for rapid laboratory diagnosis of Candida species in clinical samples [Table-4].

Table 4- Antifungal testing sensitivity pattern of isolated Candida

spp.

\begin{tabular}{lcccc|} 
Species & Fluconazole & Clotrimazole & Amphotericin-B & Nystain \\
\hline Candida albicans & $22(100 \%)$ & $18(81.8 \%)$ & $21(95.4 \%)$ & $22(100 \%)$ \\
Candida glabrata & $0(0 \%)$ & $0(100 \%)$ & $8(100 \%)$ & $8(100 \%)$ \\
Candida krusei & $0(0 \%)$ & $0(100 \%)$ & $5(100 \%)$ & $5(100 \%)$ \\
Candia tropicalis & $4(100 \%)$ & $3(75 \%)$ & $4(100 \%)$ & $4(100 \%)$ \\
Candida parapsilosis & $1(100 \%)$ & $1(100 \%)$ & $1(100 \%)$ & $1(100 \%)$ \\
\hline
\end{tabular}

In this study fluconazole was effective for C. albicans. Nystain \& Amphotericin B approximate $95-100 \%$ sensitive for all isolated species of Candida.

Out of total 100 women, 17 were diabetic. Out of them 12(70.58\%) had vulvovaginal candidiasis. 3 were HIV reactive and out of them 1 (33.3\%) had vulvovaginal candidiasis.

\section{Discussion}

Candidal vulvovaginitis or vaginal thrush is an infection of the vagina's mucous membranes by Candida albicans. Up to $75 \%$ of women will have this infection at some point in their lives, and approximately $5 \%$ will have recurring episodes. It is the second most common cause of vaginal inflammation after bacterial vaginosis-CDC. It is most commonly caused by a type of fungus known as Candida albicans. The Candida species of fungus is found naturally in the vagina, and is usually harmless. However, if the conditions in the vagina change, Candida albicans can cause the symptoms of 
thrush. Symptoms of thrush can also be caused by Candida glabrata, Candida krusei, Candida parapsilosis, and Candida tropicalis. Non-albicans Candida is commonly found in complicated cases of vaginal thrush such that first line treatment is ineffective. These cases are more likely in immunocompromised patients-CDC.

Jorizzo [8] and Sobel, et al [9] has observed that vulvovaginitis patients presented with white curdy vaginal discharge which is comparable to our study. In a large study of women attending a sexually transmitted disease clinic, only $28 \%$ of 545 women with pruritis, burning or a vaginal discharge were $C$. albicans culture positive. Thus, in symptomatic women, a positive wet mount or culture for Candida is necessary to assess whether this organism is present in the vagina. The findings in the our study shows association between a positive Candida culture \& symptomatology along with current oral contraceptive and antibiotic usage and pregnancy parallel earlier reports [10-12].

The swabs were taken \& were inoculated into Sabouraud's Dextrose Agar and Hichrom Candida agar $[5,6]$. The White to cream colored, pasty, smooth colonies were identified and confirmed by wet mount, Gram's staining, Germ tube testing and Dalmau plate (cornmeal agar) morphology, Sugar assimilation and fermentation tests. The isolates identified by biochemical tests were also compared with growth and color changes in Hichrom Candida agar. $[5,6]$. Antifungal sensitivity pattern of isolates were performed by Modified Kirby Bauer Disc diffusion technique on Muller Hinton agar $+2 \%$ glucose + Methylene blue [7]. In our study, a total number of 100 patients of vaginitis were included, among them 45 samples indicates positivity, out of them 31 samples indicate Candida albicans and other are non-albicans species. Thus Out of total 45 Candida positive specimens $31(68.88 \%)$ specimens were of Candida albicans, 1 was of (2.22\%) Candida parapsilosis, 8 was of Candida glabrata (17.77\%), 2 was of Candida krusei (4.4\%), Candia tropicalis was $3(6.66 \%)$. Candida albicans was the most common species isolated $(77.5 \%)$ \& C. glabrata $(10.6 \%)$, C. tropicalis $(3.8 \%), C$. krusei $(2.7 \%)$ on rapid identification on chrom agar inoculation. Prevalence and susceptibility to Candida spp. with fluconazole suggested that yeasts were isolated in $111(18.5 \%)$ women and these consisted of C. albicans (39, 35.1\%), Candida glabrata (56, 50.4\%), C. tropicalis (12, 10.8\%), C. krusei $(3,2.7 \%)$ and C. parapsilosis (1, $0.9 \%$ ). Multiple epidemiologic studies have indicated that Candida albicans is responsible for in excess of $90 \%$ of episodes of VVC, although rare reports indicate that non- albicans Candida (NAC) species are responsible for $10-30 \%$ of episodes in certain geographic regions [13-15].

In our study most of the patients belongs to age group 21-30 (59.32\%). In a study of clinical pattern of Candidiasis infections in Bombay, Dalal and Kelkar [16] had noticed more number of patients belonging to the age group of $21-40$ years (35\%). Clayton and Noble [17] in a study on observations on the epidemiology of Candida albicans found majority of patients belonging to the age group of 20 -60 years.

Montes, et al [18] and Macneill \& Carey [19] have found diabetes mellitus as one of the common predisposing factors for Candidiasis of various types which is also seen in this study. It was found that $11(20.4 \%)$ of 54 Candidal cases, had diabetes as the risk factor.

Talib \& Singh [20] and Baradkar \& Karyakarte [21] have observed positive culture in $65 \%$ and $63.33 \%$ respectively, all of whom were HIV positive patients.
In Our study Hichrom agar was found to be more sensitive in comparison to conventional culture and identification tests. In studies by Odds and Bernaerts [22], Louwagie, et al [23] and Moyer, et al [24], a major advantage of Chrom agar was the ability to detect mixed cultures of yeasts in clinical specimens. In all three previous studies, Chrom agar was superior to other routine and selective media in detecting multiple Candida species in both clinical and stock cultures. Our results were in agreement with these prior studies.

\section{Conclusion}

Vaginal candidiasis is an extremely common infection in $60-70 \%$ women during their reproductive age at least once in their lives $[1,2]$. Epidemiological profile of genitourinary candidiasis varies globally depending upon socioeconomic and health factors. The associated risk factors in pregnancy are - poor socioecomic strata \& poor hygiene, late pregnancy, multigravida, Immunocompromised status like presence diabetes mellitus and HIV infection [15]. There is an increase in infections with non-albicans Candida spp. and few of them are intrinsically resistant to azoles like $C$. krusei. This specifies the need of species identification and antifungal susceptibility as a part of the laboratory diagnosis of vaginal candidiasis. Effective antifungal treatment is an important criterion in treating the candidial infections [25].

\section{Conflict of Interest : None declared.}

\section{References}

[1] Geiger A.M., Foxman B. \& Gillespie B.W. (1995) Am. J. Public Health, 85, 1146-1148.

[2] Sobel J.D. (1993) Clin. Obstet. Gynecol., 36, 153-165.

[3] Jovanovic R., Congema E. \& Nguyen H.T. (1991) J. Repro. Med., 36, 593-597.

[4] Fauci A.S. (1997) Harrison's Principles of Internal Medicine, 14th ed.(1), 805.

[5] Pfaller M.A., Houston A., Coffmann S. (1996) J. Clin. Microbiol., $34,58-61$.

[6] CLSI (2009) Methods of Antifungal Susceptibility Testing of Yeast, Approved guidelines, 29(17), M44-A2.

[7] Houang E.T., Chu K.C., Koehler A.P., Cheng A.F. (1997) J. Clin. Pathol., 50, 563-565.

[8] Jorizzo J.L. (1984) Dermatol. Clin., 2, 19-27.

[9] Sobel J.D., Faro S., Force R.W., Foxman B., Ledger W.J., Nyirjesy P.R., Reed B.D., Summers P.R. (1998) Am. J. Obstet. Gynecol., 178, 203-211.

[10]Spinillo A., Capuzzo F., Nicola S. (1995) Am. J. Obstet. Gynecol., 51, 293-297.

[11]Morton R.S., Rashid S. (1977) Proc. R. Soc. Med., (4), 3-6.

[12]Ryley J.F. (1986) J. Med. Vet. Mycology, 4, 5-22.

[13]Sobel J.D. (2007) Lancet, 369, 1961-1971.

[14]Vermitsky J.P., Self M.J., Chadwick S.G. (2008) J. Clin. Microbiol., 46, 1501-1503.

[15]Mohanty S., Xess I., Hasan F. (2007) Indian J. Med. Res., 126, 216-219.

[16]Dalal P.J., Kelkar S.S. (1980) Ind. J. Der. Ven. Lep., 46(1), $31-$ 32.

[17]Clayton Y.M., Noble W. (1966) J. Clin. Path., 19, 76-78. 
Microbiological Study of Various Candida Species and Its Antifungal Sensitivity Testing Isolated from Antenatal Women with Vaginitis, in Tertiary Care Teaching Hospital, Western India

[18]Montes L.F., Moschella S.L., Hurley H.J. (1992) Text Book of Dermatology, 3rd ed.(1), 913-923.

[19]Witkin S.S., Jeremias J., Ledger W.J. (1998) J. Allergy Clin. Immunol., 81, 412-416.

[20]Talib S.H., Singh J. (1997) Indian J. Pathol. Microbiol., 40(3), 377-382.

[21]Baradkar V.P., Karyakarte R.P. (1999) Indian J. Med. Microbiol., 17(1), 42-44.

[22] Odds F.C., Bernaerts R. (1994) J. Clin. Microbio., 32, 19231929.

[23]Louwagie B., Surmont I., Verhaegen J., Odds F. (1995) Eur. J. Clin. Microbiol. Infect. Dis., 14, 406-411.

[24]Veena M., Vidya G.S., Sharma A., Prakash M.R. (2012) Int. J. Biol. Med. Res., 3(2), 1778-1782.

[25]Babin D., Kotigadde S., Rao S., Rao T.V. (2013) Int. J. Biol. Med. Res., 3(1), 55-59. 